\title{
Рост внутренних оптических потерь с увеличением тока накачки и выходная мощность лазеров на квантовых ямах
}

\author{
() 3.Н. Соколова ${ }^{1}$, Д.А. Веселов ${ }^{1}$, Н.А. Пихтин ${ }^{1}$, И.С. Тарасов $^{1}$, Л.В. Асрян ${ }^{2}$ \\ ${ }^{1}$ Физико-технический институт им. А.Ф. Иофффе Российской академии наук, \\ 194021 Санкт-Петербург, Россия \\ ${ }^{2}$ Virginia Polytechnic Institute and State University, \\ Blacksburg, VA 24061, USA \\ E-mail: zina.sokolova@mail.ioffe.ru, asryan@vt.edu
}

(Получена 19 января 2017 г. Принята к печати 25 января 2017 г.)

Представлены результаты расчетов рабочих характеристик полупроводниковых лазеров на квантовых ямах с учетом роста внутренних оптических потерь в волноводной области с увеличением тока накачки. Использовано условие глобальной электронейтральности в структуре, которое заключается в равенстве суммарного заряда электронов в активной и волноводной областях суммарному заряду дырок в этих двух областях. Получено хорошее согласие измеренной и рассчитанной ватт-амперных характеристик.

DOI: $10.21883 /$ FTP.2017.07.44661.8522

\section{1. Введение}

Повышение оптической мощности планарных полупроводниковых лазеров инфракрасного диапазона с наноразмерной активной областью все еще остается актуальной задачей, несмотря на достигнутые в этой области успехи. Одной из причин ограничения мощности является возрастание внутренних оптических потерь в лазерной структуре при увеличении тока накачки. Эта проблема исследовалась в различных работах. В частности, в работах [1-6] теоретически изучалось влияние роста внутренних оптических потерь с увеличением тока накачки на рабочие характеристики лазеров с квантоворазмерными активными областями. Нами недавно были выполнены экспериментальные исследования этой проблемы в лазерных структурах с длиной волны излучения $\sim 1$ мкм [7-10]. В работе [9] была разработана специальная методика измерения коэффициента поглощения в слоях полупроводникового лазера при импульсной токовой накачке.

В современных полупроводниковых лазерах низкоразмерная активная область окружена более широкозонным объемным материалом - волноводной областью (областью оптического ограничения - optical confinement layer, OCL). Инжектированные из эмиттеров электроны и дырки сначала попадают в волноводную область, а далее с конечной скоростью захватываются в активную область [11]. Таким образом, захват носителей из волноводной области лазера в активную область не является мгновенным процессом.

При расчетах характеристик полупроводниковых лазеров, начиная со времен использования объемных (трехмерных) активных областей, принято считать, что концентрации электронов и дырок в активной области равны друг другу [12], т.е. в активной области имеет место локальная электронейтральность. Условие локальной электронейтральности может перестать быть справедливым для квантово-размерных активных областей - концентрации носителей в активной области могут сильно различаться [13]. Таким образом, при расчетах характеристик полупроводниковых лазеров является более обоснованным использование условия глобальной электронейтральности, которое заключается в равенстве суммарного заряда электронов в активной и волноводной областях суммарному заряду дырок в этих двух областях $[14,15]$.

В настоящей работе мы изучаем влияние роста внутренних оптических потерь в волноводной области при увеличении тока накачки на характеристики полупроводниковых лазеров с квантовыми ямами (КЯ). Мы используем здесь условие глобальной электронейтральности и учитываем немгновенность захвата носителей в квантовые ямы. В [1-3] для построения аналитической модели использовалось условие локальной электронейтральности, а в [4-6] не учитывалась конечность скорости захвата носителей заряда в ямы.

\section{2. Теоретическая модель}

Для расчета лазерных характеристик мы использовали пять стационарных скоростных уравнений [16]: для свободных электронов в объемной волноводной области толщиной $b\left[b\left(\partial n^{\mathrm{OCL}} / \partial t\right)=0\right]$,

$$
\begin{aligned}
\frac{j}{e}+N_{\mathrm{QW}} \frac{n^{\mathrm{QW}}}{\tau_{n, \text { esc }}} & -N_{\mathrm{QW}} v_{n, \text { capt }, 0}\left(1-f_{n}\right) n^{\mathrm{OCL}} \\
& -b B_{3 \mathrm{D}} n^{\mathrm{OCL}} p^{\mathrm{OCL}}=0,
\end{aligned}
$$

для свободных дырок в объемной волноводной области $\left[b\left(\partial p^{\mathrm{OCL}} / \partial t\right)=0\right]$,

$$
\begin{aligned}
\frac{j}{e}+N_{\mathrm{QW}} \frac{p^{\mathrm{QW}}}{\tau_{p, \text { esc }}} & -N_{\mathrm{QW}} v_{p, \text { capt }, 0}\left(1-f_{p}\right) p^{\mathrm{OCL}} \\
& -b B_{3 \mathrm{D}} n^{\mathrm{OCL}} p^{\mathrm{OCL}}=0,
\end{aligned}
$$


для электронов, локализованных в КЯ $\left(\partial n^{\mathrm{QW}} / \partial t=0\right)$,

$$
\begin{gathered}
v_{n, \text { capt }, 0}\left(1-f_{n}\right) n^{\mathrm{OCL}}-\frac{n^{\mathrm{QW}}}{\tau_{n, \mathrm{esc}}}-B_{2 \mathrm{D}} n^{\mathrm{QW}} p^{\mathrm{QW}} \\
-v_{g} g^{\max }\left(f_{n}+f_{p}-1\right) \frac{N}{S}=0,
\end{gathered}
$$

для дырок, локализованных в КЯ ( $\left.\partial p^{\mathrm{QW}} / \partial t=0\right)$,

$$
\begin{gathered}
v_{p, \text { capt }, 0}\left(1-f_{p}\right) p^{\mathrm{OCL}}-\frac{p^{\mathrm{QW}}}{\tau_{p, \text { esc }}}-B_{2 \mathrm{D}} n^{\mathrm{QW}} p^{\mathrm{QW}} \\
-v_{g} g^{\max }\left(f_{n}+f_{p}-1\right) \frac{N}{S}=0,
\end{gathered}
$$

и для фотонов в лазерной моде $(\partial N / \partial t=0)$,

$$
v_{g} N_{\mathrm{QW}} g^{\max }\left(f_{n}+f_{p}-1\right) N-v_{g}\left(\beta+\alpha_{\text {int }}\right) N=0 .
$$

Неизвестные величины в уравнениях $(1)-(5): n^{\mathrm{OCL}}$ и $p^{\text {OCL }}$ - трехмерные концентрации свободных электронов и дырок в волноводной области, $n^{\mathrm{QW}}$ и $p^{\mathrm{QW}}-$ двумерные концентрации электронов и дырок, локализованных в КЯ, $N$ - число фотонов стимулированного излучения, $f_{n}$ и $f_{p}$ - степени заполнения (заселенности) состояний, соответствующих нижнему краю электронной и верхнему краю дырочной подзон.

Степени заполнения $f_{n}$ и $f_{p}$ связаны с двумерными концентрациями электронов и дырок в КЯ, $n^{\mathrm{QW}}$ и $p^{\mathrm{QW}}$, следующим образом [1,2,17]:

$$
f_{n}=1-\exp \left(-\frac{n^{\mathrm{QW}}}{N_{c}^{2 \mathrm{D}}}\right), f_{p}=1-\exp \left(-\frac{p^{\mathrm{QW}}}{N_{v}^{2 \mathrm{D}}}\right),
$$

где $N_{c, v}^{2 \mathrm{D}}=m_{e, h h}^{\mathrm{QW}} T /\left(\pi \hbar^{2}\right)-$ двумерные эффективные плотности состояний в зоне проводимости и валентной зоне в КЯ, $m_{e, h h}^{\mathrm{QW}}$ - эффективные массы электронов и дырок в КЯ, $T$ - температура в энергетических единицах.

В уравнения (1)-(5) входят следующие параметры: $j$ - плотность тока инжекции, $e-$ заряд электрона, $N_{\mathrm{QW}}$ - число идентичных, т.е. имеющих одну и ту же ширину и одинаковый состав, КЯ, $\tau_{n \text {, esc }}$ и $\tau_{p \text {, esc }}-$ времена термических выбросов электронов и дырок из КЯ в волноводную область, $v_{n \text {, capt }, 0}$ и $v p$, capt, $0-$ скорости захвата (capture velocities) электронов и дырок в пустую (при $f_{n}=0$ и $f_{p}=0$ ) одиночную КЯ, измеряемые в $\mathrm{cM} / \mathrm{c} ; B_{3 \mathrm{D}}$ и $B_{2 \mathrm{D}}-$ коэффициенты спонтанной излучательной рекомбинации в объемной (волноводной) области и в двумерной области (КЯ), измеряемые в см $3 / \mathrm{c}$ и $\mathrm{cm}^{2} /$ с соответственно (см. [18] и [19] для выражений для $B_{3 \mathrm{D}}$ и $\left.B_{2 \mathrm{D}}\right) ; v_{g}$ - групповая скорость света; $g_{\max }-$ максимальный коэффициент усиления в каждой КЯ; $S=W L, W$ - ширина полоскового контакта, $L-$ длина резонатора Фабри-Перо; $\beta=(2 L)^{-1} \ln \left(R_{1} R_{2}\right)^{-1}-$ потери, связанные с выходом излучения из резонатора, $R_{1}, R_{2}$ - коэффициенты отражения зеркал лазера с резонатором Фабри-Перо; $\alpha$ int - коэффициент внутренних оптических потерь в лазерной структуре.
Времена термических выбросов электронов и дырок из КЯ в волноводную область даются согласно $[16,20]$

$$
\begin{aligned}
\tau_{n, \mathrm{esc}} & =\frac{1}{v_{n, \mathrm{capt}, 0}\left(1-f_{n}\right)} \frac{N_{c}^{2 \mathrm{D}}}{n_{1}}, \\
\tau_{p, \mathrm{esc}} & =\frac{1}{v_{p, \mathrm{capt}, 0}\left(1-f_{p}\right)} \frac{N_{v}^{2 \mathrm{D}}}{p_{1}},
\end{aligned}
$$

где

$$
\begin{aligned}
& n_{1}=N_{c}^{3 \mathrm{D}} \exp \left(-\frac{\Delta E_{c}-\varepsilon_{n}^{\mathrm{QW}}}{T}\right), \\
& p_{1}=N_{v}^{3 \mathrm{D}} \exp \left(-\frac{\Delta E_{v}-\varepsilon_{p}^{\mathrm{QW}}}{T}\right),
\end{aligned}
$$

$N_{c, v}^{3 \mathrm{D}}=2\left[m_{c, v}^{\mathrm{OCL}} T /\left(2 \pi \hbar^{2}\right)\right]^{3 / 2}-$ трехмерные эффективные плотности состояний в зоне проводимости и в валентной зоне волноводной области, $m_{c, v}^{\mathrm{OCL}}-$ эффективные массы электронов и дырок в волноводной области, $\Delta E_{c, v}-$ разрывы краев зоны проводимости и валентной зоны на гетероконтакте КЯ и волноводной области, $\varepsilon_{n}^{\mathrm{QW}}$ и $\varepsilon_{p}^{\mathrm{QW}}-$ энергии соответственно нижнего и верхнего краев электронной и дырочной подзон размерного квантования в КЯ.

В уравнения (1)-(4) в качестве параметров входят

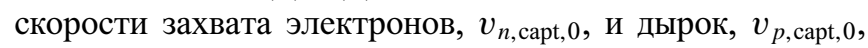
из волноводной области в пустую КЯ. Скорости захвата являются характеристиками КЯ и зависят от ее ширины и глубины, т.е. зависят от состава материалов КЯ и окружающих ее слоев. Величины скоростей захвата различаются в различных лазерных структурах. В работах $[21,22]$ были определены скорости захвата электронов в пустую одиночную КЯ в реальных лазерных структурах. Для этого в этих работах использовались экспериментальные лазерные характеристики и теоретические расчеты.

В данной работе скорости захвата электронов и дырок в пустую КЯ считались одинаковыми и равными $5 \cdot 10^{5} \mathrm{~cm} / \mathrm{c}$. При этом скорости захвата в заполненную КЯ [16],

$$
v_{n, \mathrm{capt}}=v_{n, \mathrm{capt}, 0}\left(1-f_{n}\right), v_{p, \mathrm{capt}}=v_{p, \mathrm{capt}, 0}\left(1-f_{p}\right),
$$

согласно формулам (6) оказываются различными. В работе [15] показано, что характеристики исследуемых лазеров сильно зависят от скорости захвата электронов и очень слабо от скорости захвата дырок.

В расчетах мы использовали условие глобальной электронейтральности, которое заключается в равенстве суммарного заряда электронов в волноводной области $\left(n^{\mathrm{OCL}}\right)$ и в КЯ $\left(n^{\mathrm{QW}}\right)$ суммарному заряду дырок в этих двух областях:

$$
e\left(N_{\mathrm{QW}} n^{\mathrm{QW}}+b n^{\mathrm{OCL}}\right)=e\left(N_{\mathrm{QW}} p^{\mathrm{QW}}+b p^{\mathrm{OCL}}\right),
$$

где $b-$ ширина волноводной области. 
Параметры экспериментальной лазерной структуры

\begin{tabular}{l|c|c|c}
\hline Название слоя & Толщина слоя, мкм & Материал слоя & Легирование слоя \\
\hline Подложка & 100 & $\mathrm{GaAs}$ & $N, 1 \cdot 10^{18} \mathrm{~cm}^{-3}$ \\
Эмиттер & 1.5 & $\mathrm{Al}_{0.1} \mathrm{Ga}_{0.9} \mathrm{As}$ & $n, 50^{17} \mathrm{~cm}^{-3}$ \\
Волновод & 1.73 & $\mathrm{GaAs}$ & $\mathrm{cm}$ \\
Волновод & 0.2 & $\mathrm{GaAs}$ & легирован \\
Квантовая яма & 0.009 & $\mathrm{InGaAs}$ & легирован \\
Спейсер & 0.03 & $\mathrm{GaAs}$ & не легирован \\
Квантовая яма & 0.009 & $\mathrm{InGaAs}$ & не легирован \\
Волновод & 0.2 & $\mathrm{GaAs}$ & не легирован \\
Волновод & 1 & $\mathrm{GaAs}$ & $p, 5 \cdot 10^{16} \mathrm{~cm}^{-3}$ \\
Эмиттер & 1 & $\mathrm{Al}_{0.3} \mathrm{Ga} 0.7$ & $P, 1 \cdot 10^{18} \mathrm{~cm}^{-3}$ \\
Контактный слой & 0.3 & $\mathrm{GaAs}$ & $P, 3 \cdot 10^{18} \mathrm{~cm}^{-3}$
\end{tabular}

\section{3. Обсуждение характеристик лазера}

Система уравнений (1)-(5), дополненная условием (10), решалась численно для лазерной структуры, длина волны излучения которой при комнатной температуре составляла 1.075 мкм. Лазерная структуpa AlGaAs/GaAs/InGaAs была выращена на подложке GaAs (таблица). Структура содержит две напряженные квантовые ямы $\operatorname{In}_{0.31} \mathrm{Ga}_{0.69} \mathrm{As}$ толщиной $90 \AA$. Материалом волновода шириной $b=3.2$ мкм является GaAs, материалом эмиттеров - AlGaAs. Длина резонатора Фабри-Перо $L=5.1$ мм, ширина полоскового контакта $W=100$ мкм, коэффициент отражения обоих зеркал $R=0.05$, потери на выход излучения $\beta=5.87 \mathrm{~cm}^{-1}$. Параметры резонатора исследуемого лазера позволяют экспериментально исследовать рост внутренних оптических потерь по методике, изложенной в [9]. Выходная оптическая мощность такого лазера измерялась и рассчитывалась с обоих зеркал резонатора. Как было показано в работе [8], лазеры с двумя просветленными зеркалами имеют более близкую к линейной ватт-амерную характеристику (BTAX) по сравнению с обычными лазерами за счет более слабого влияния внутренних оптических потерь на выходную мощность. Максимальный модальный коэффициент усиления в одной КЯ $g^{\max }=36.5 \mathrm{~cm}^{-1}$.

В настоящей работе мы учли возрастание внутренних оптических потерь при увеличении плотности тока накачки. В случае достаточно широкой волноводной области (3.2 мкм) электромагнитная волна распространяется практически полностью в волноводной области, в которой и происходят оптические потери. В слаболегированных полупроводниках (волноводная область нашей структуры не легирована) коэффициент внутренних оптических потерь линейно зависит от концентраций носителей заряда [12],

$$
\begin{gathered}
\alpha_{\mathrm{int}}=\alpha_{0}+\alpha^{\mathrm{OCL}}, \alpha^{\mathrm{OCL}}=\alpha_{n}+\alpha_{p}, \\
\alpha_{n}=\Gamma^{\mathrm{OCL}} \sigma_{n} n^{\mathrm{OCL}}, \alpha_{p}=\Gamma^{\mathrm{OCL}} \sigma_{p} p^{\mathrm{OCL}},
\end{gathered}
$$

где $\alpha^{\mathrm{OCL}}-$ коэффициент внутренних оптических потерь в волноводной области, $\sigma_{n}$ и $\sigma_{p}-$ сечения поглощения излучения свободными электронами и дырками в волноводной области, $\Gamma^{\mathrm{OCL}}-$ фактор оптического ограничения в волноводной области, $\alpha_{0}-$ постоянная компонента коэффициента внутренних оптических потерь, связанная в первую очередь с поглощением излучения в эмиттерах.

Ввиду немгновенности захвата носителей заряда из волноводной области в квантовые ямы концентрации этих носителей в волноводной области растут с увеличением тока инжекции в режиме лазерной генерации $[1-4,14,16]$. Вследствие этого, как видно из (12), происходит увеличение внутренних оптических потерь в волноводной области лазерной структуры.

Мы использовали для сечений поглощения свободными носителями в GaAs при комнатной температуре и при энергиях фотонов, близких к ширине запрещенной зоны GaAs, следующие значения: $\sigma_{n}=3 \cdot 10^{-18} \mathrm{~cm}^{2}$ и $\sigma_{p}=7 \cdot 10^{-18} \mathrm{~cm}^{2}[12]$; при этом $\Gamma^{\mathrm{OCL}}=0.9843$. Для того чтобы расчетное и экспериментальное значения мощности $P$ совпадали при плотности тока $j=1$ кА/см ${ }^{2}$, бралось $\alpha_{0}=0.35 \mathrm{~cm}^{-1}$.

Численно решив систему уравнений (1)-(5), дополненную условием глобальной электронейтральности (10), мы получили зависимости следующих величин от плотности тока накачки: а) концентраций электронов и дырок в КЯ, б) концентраций электронов и дырок в волноводной области, в) внутренних оптических потерь, г) заселенностей электронной и дырочной подзон размерного квантования в КЯ, а также д) мощности выходного оптического излучения.

Рассчитанные зависимости концентраций электронов и дырок в одной КЯ от плотности тока накачки представлены на рис. 1. Из рисунка видно, что в активной области рассматриваемой лазерной структуры концентрации электронов и дырок сильно различаются. На пороге генерации концентрация электронов превышает концентрацию дырок в 1.5 раза, a с возрастанием плотности тока накачки концентрация дырок начинает превосходить концентрацию электронов. При $j=20$ кА $/ \mathrm{cm}^{2}$ концентрация дырок уже в 5 раз больше, чем концентрация электронов. Это означает, что в активной области существует „недостаток“ электронов и, следовательно, нет локальной электронейтральности 


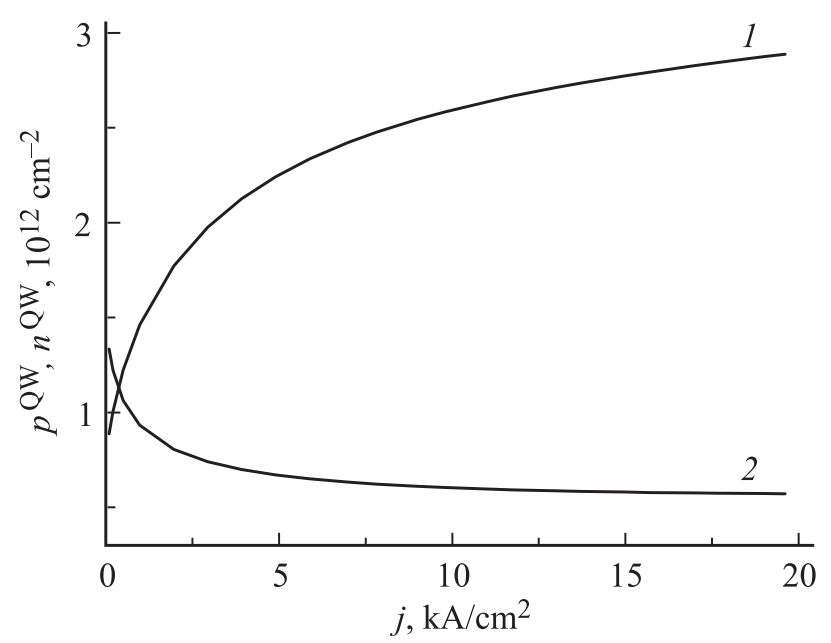

Рис. 1. Концентрации дырок (1) и электронов (2) в одной квантовой яме в зависимости от плотности тока накачки.

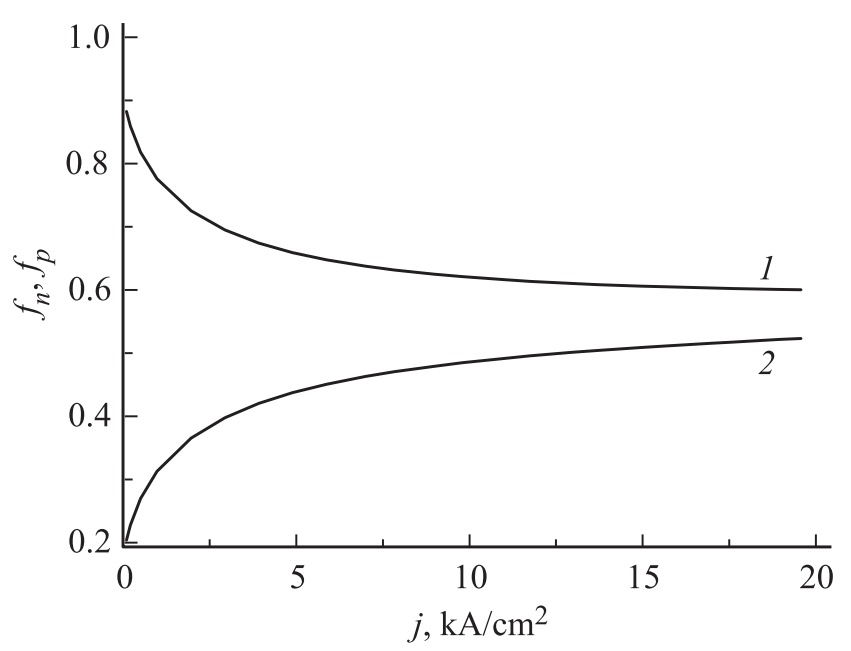

Рис. 2. Заселенность состояний, соответствующих нижнему краю электронной подзоны $(1)$ и верхнему краю дырочной подзоны (2) размерного квантования в КЯ, в зависимости от плотности тока накачки.

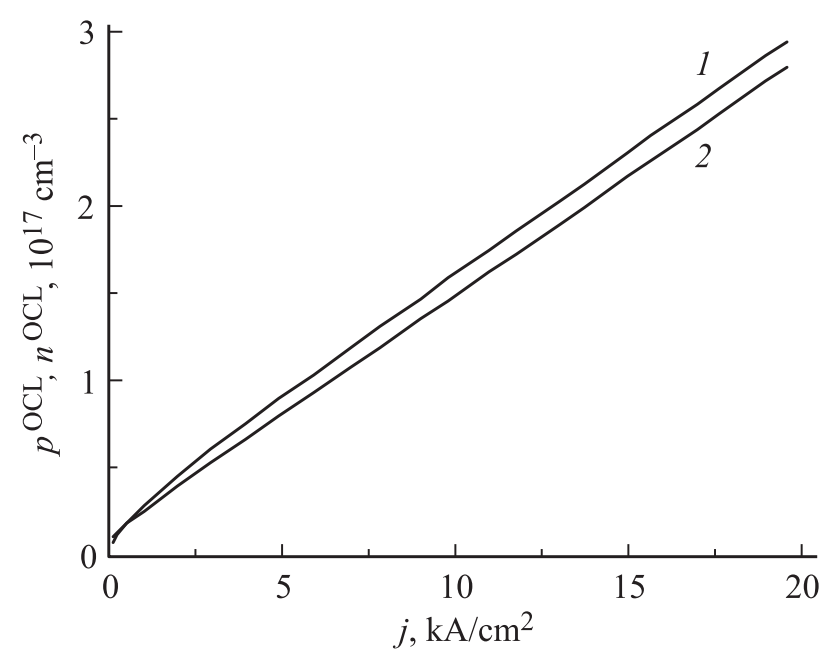

Рис. 3. Концентрации электронов (1) и дырок (2) в волноводной области в зависимости от плотности тока накачки.

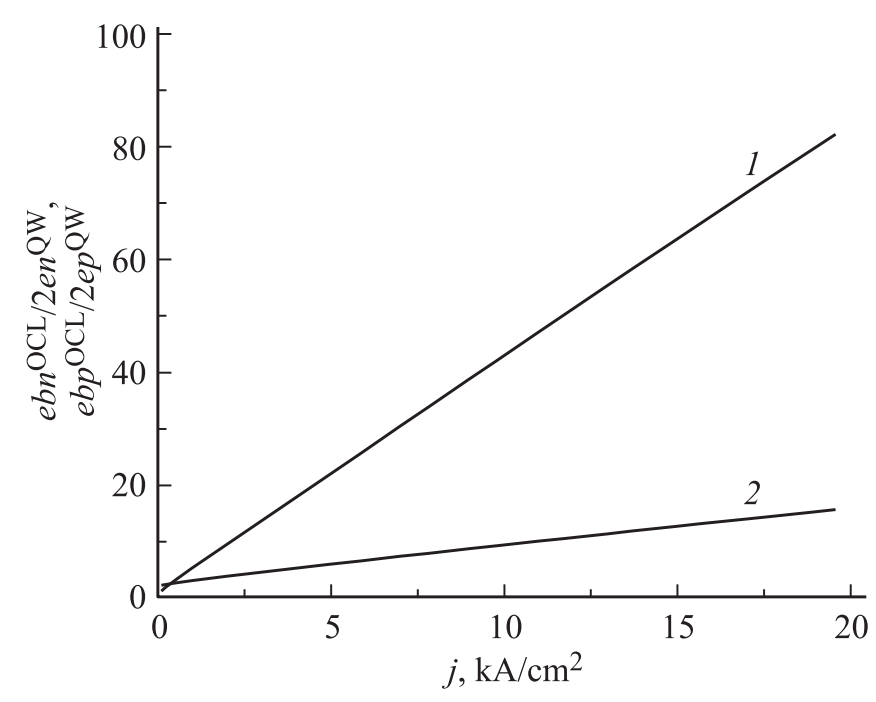

Рис. 4. Отношение заряда электронов в волноводной области к заряду электронов в КЯ (1) и отношение заряда дырок в волноводной области к заряду дырок в КЯ (2) в зависимости от плотности тока накачки.

электронов и дырок. Это выражается в уменьшении степени заполнения нижнего края электронной подзоны размерного квантования в КЯ и увеличении степени заполнения верхнего края дырочной подзоны с ростом плотности тока накачки (рис. 2).

На рис. 3 приведены концентрации электронов и дырок в волноводной области. Как видно из рисунка, эти концентрации мало отличаются друг от друга. Даже при $j=20$ кА $/ \mathrm{cm}^{2}$ концентрация электронов превышает концентрацию дырок всего в 1.05 раза. Следует отметить, что заряд каждого знака (как электронов, так и дырок) в волноводной области существенно больше, чем соответствующий заряд в двух квантовых ямах (рис. 4). При $j=20$ кА/см ${ }^{2}$ заряд дырок в волноводной области в 15 раз больше, чем в КЯ, а заряд электронов в волноводной области в 82 раза превосходит заряд электронов в КЯ.

Таким образом, при увеличении плотности тока накачки происходит накопление носителей заряда в волноводной области. Это, согласно выражениям (11) и (12), приводит к росту внутренних оптических потерь в волноводной области с увеличением плотности тока накачки (рис. 5, кривая 1). На рис. 5 также приведены зависимости от плотности тока накачки внутренних оптических потерь в волноводной области, обусловленных поглощением света дырками, $\alpha_{p}$ (кривая 2), и поглощением света электронами, $\alpha_{n}$ (кривая 3). При рассмотренных значениях плотности инжекционного тока, которые соответствуют экспериментальному диапазону токов накачки в исследованной лазерной структуре (таблица), концентрации носителей и внутренние оптические потери в волноводной области растут практически линейно с увеличением плотности тока (рис. 3 и 5).

Возрастание внутренних оптических потерь приводит к ослаблению роста концентрации фотонов в резонаторе 




Рис. 5. Коэффициент внутренних оптических потерь в волноводной области (1), „вклад“, обусловленный поглощением света дырками (2), электронами (3), в зависимости от плотности тока накачки.

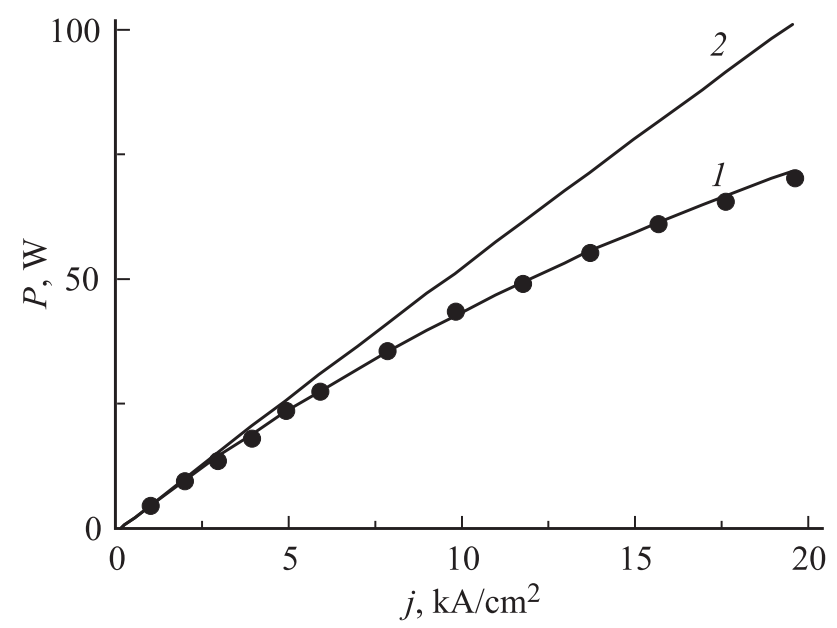

Рис. 6. Оптическая мощность лазера $(P)$ в зависимости от плотности тока накачки (ватт-амперная характеристика). Сплошные кривые - расчет: с учетом зависимости внутренних оптических потерь от тока накачки $(1)$, для $\alpha_{\text {int }}=$ const (2); точки - эксперимент.

лазера при увеличении плотности тока накачки. Это, в свою очередь, уменьшает мощность оптического излучения, выходящую из лазера:

$$
P(j)=\frac{\hbar \omega}{e} S j_{\mathrm{stim}}(j) \frac{\beta}{\beta+\alpha_{\mathrm{int}}},
$$

где

$$
j_{\text {stim }}=e v_{g}\left(\beta+\alpha_{\text {int }}\right) \frac{N}{S}
$$

- плотность тока стимулированной излучательной рекомбинации в двух квантовых ямах [16]. Таким образом, возрастание внутренних оптических потерь с увеличением тока накачки приводит к ограничению роста выходного излучения лазера.
Ватт-амперные характеристики лазера приведены на рис. 6. Сплошные кривые представляют ВтАХ, рассчитанные с учетом возрастания внутренних оптических потерь в волноводной области лазера (кривая 1) и при постоянных внутренних оптических потерях $\alpha_{\text {int }}=0.5 \mathrm{~cm}^{-1}$ (кривая 2). При $j=20$ кА/см ${ }^{2}$ значения мощности на кривых 1 и 2 различаются в 1.4 раза. Точками на рис. 6 изображены экспериментальные значения ВТАХ, измеренные в настоящей работе для лазерной структуры AlGaAs/GaAs/InGaAs (таблица). Как видно из рисунка, имеет место очень хорошее согласие экспериментальной и теоретической ВтАХ, рассчитанной с учетом возрастания оптических потерь с увеличением тока накачки. Следует отметить, что для совпадения рассчитанной мощности с экспериментальным значением при $j=1 \kappa \mathrm{A} / \mathrm{cm}^{2}$ постоянная компонента $\alpha_{0}$ коэффициента внутренних оптических потерь (см. выражение (11)) была положена в наших расчетах равной $0.35 \mathrm{~cm}^{-1}$.

\section{4. Заключение}

Представлены результаты расчетов рабочих характеристик полупроводникового лазера на основе структуры $\mathrm{AlGaAs} / \mathrm{GaAs} / \mathrm{InGaAs}$ с двумя КЯ. Учтен рост внутренних оптических потерь в волноводной области структуры с увеличением плотности тока накачки в режиме лазерной генерации (рис. 5). Использовано условие глобальной электронейтральности в структуре (выражение (10)), заключающееся в равенстве суммарного заряда электронов в волноводной области и в двух квантовых ямах суммарному заряду дырок в этих областях.

Получено хорошее согласие измеренной и рассчитанной ватт-амперных характеристик (рис. 6). Совпадение рассчитанной и экспериментальной ВтАХ подтверждает необходимость учета возрастания внутренних оптических потерь в волноводной области лазерной структуры при увеличении тока накачки.

Для уменьшения насышения ВтАХ необходимо уменьшить внутренние оптические потери в волноводной области лазера, для чего необходимо понизить концентрации носителей заряда в этой области. Для этого можно, например, использовать лазерные структуры с несколькими квантовыми ямами. Меньшие концентрации в волноводной области могут быть также достигнуты с использованием структур с нелегированной волноводной областью, структур с оптимизированной шириной волноводной области [18], а также структур с большей скоростью захвата носителей из волноводной области в квантовые ямы [15].

Работа выполнена в соответствии с Государственной программой ФТИ им. А.Ф. Иоффе. Л.В. Асрян благодарит Исследовательский офис армии CША (U.S. Army Research Office, грант № W911NF-13-1-0445) за поддержку данной работы. 


\section{Список литературы}

[1] L.V. Asryan, S. Luryi. Appl. Phys. Lett., 83, 5368 (2003).

[2] L.V. Asryan, S. Luryi. IEEE J. Quant. Electron., 40, 833 (2004).

[3] L.V. Asryan. Appl. Phys. Lett., 88, 073107 (2006).

[4] E.A. Avrutin, B.S. Ryvkin. Semicond. Sci. Technol., 32, 015004 (2017).

[5] B.S. Ryvkin, E.A. Avrutin. J. Appl. Phys., 97, 123103 (2005).

[6] B.S. Ryvkin, E.A. Avrutin. Electron. Lett., 42, 1283 (2006).

[7] Д.А. Веселов, В.А. Капитонов, Н.А. Пихтин, А.В. Лютецкий, Д.Н. Николаев, С.О. Слипченко, З.Н. Соколова, В.В. Шамахов, И.С. Шашкин, И.С. Тарасов. Квант. электрон., 44 (11), 993 (2014).

[8] Д.А. Веселов, Н.А. Пихтин, А.В. Лютецкий, Д.Н. Николаев, С.О. Слипченко, 3.Н. Соколова, В.В. Шамахов, И.С. Шашкин, В.А. Капитонов, И.С. Тарасов. Квант. электрон., 45 (7), 597 (2015).

[9] Д.А. Веселов, Н.А. Пихтин, А.В. Лютецкий, Д.Н. Николаев, С.О. Слипченко, 3.Н. Соколова, В.В. Шамахов, И.С. Шашкин, Н.В. Воронкова, И.С. Тарасов. Квант. электрон., 45 (7), 604 (2015).

[10] Д.А. Веселов, И.С. Шашкин, Ю.К. Бобрецова, К.В. Бахвалов, Н.А. Пихтин, А.В. Лютецкий, В.А. Капитонов, Н.А. Пихтин, С.О. Слипченко, 3.Н. Соколова, И.С. Тарасов. ФТП, 50 (10), 1414 (2016).

[11] L.V. Asryan, S. Luryi, R.A. Suris. Appl. Phys. Lett., 81, 2154 (2002).

[12] H.C. Casey, M.B. Panish. Heterostructure Lasers (N.Y., Academic, 1978) [Х. Кейси, М. Паниш. Лазеры на гетеponepexoдax (М., Мир, 1981)].

[13] L.V. Asryan, R.A. Suris. IEEE J. Select. Topics Quantum Electron., 3 (2), 148 (1997).

[14] Z.N. Sokolova, N.A. Pikhtin, I.S. Tarasov, L.V. Asryan. J. Phys. Conf. Ser., 740, 012002 (2016).

[15] 3.Н. Соколова, Н.А. Пихтин, И.С. Тарасов, Л.В. Асрян. Квант. электрон., 46 (9), 777 (2016).

[16] L.V. Asryan, Z.N. Sokolova. J. Appl. Phys., 115, 023107 (2014).

[17] K.J. Vahala, C.E. Zah. Appl. Phys. Lett., 52, 1945 (1988).

[18] L.V. Asryan, R.A. Suris. Semicond. Sci. Technol., 11, 554 (1996).

[19] Л.В. Асрян. Квант. электрон., 35 (12), 1117 (2005).

[20] D.-S. Han, L.V. Asryan. Nanotechnology, 21, 015201 (2010).

[21] Z.N. Sokolova, K.V. Bakhvalov, A.V. Lyutetskiy, N.A. Pikhtin, I.S. Tarasov, L.V. Asryan. Electron. Lett., 51, 780 (2015).

[22] 3.Н. Соколова, К.В. Бахвалов, А.В. Лютецкий, Н.А. Пихтин, И.С. Тарасов, Л.В. Асрян. ФТП, 50 (5), 679 (2016).

Редактор Л.В. Шаронова

\section{Internal optical loss increase with pump current and output power of quantum well lasers}

\author{
Z.N. Sokolova ${ }^{1}$, D.A. Veselov ${ }^{1}$, N.A. Pikhtin ${ }^{1}$, \\ I.S. Tarasov 1 , L.V. Asryan ${ }^{2}$ \\ ${ }^{1}$ loffe Institute, \\ 194021 St. Petersburg, Russia \\ ${ }^{2}$ Virginia Polytechnic Institute and State University, \\ Blacksburg, VA 24061, USA
}

\begin{abstract}
The calculation results of the operating characteristics of semiconductor quantum well lasers are presented, which take into account the internal optical loss increase in the waveguide region with pump current. The condition of global electroneutrality in the structure is used, which includes the charge carriers both in a two-dimensional active region (quantum well) and bulk waveguide region (optical confinement layer). A good agreement between the calculated and measured light-current characteristics is demonstrated.
\end{abstract}

